\title{
Sound Spatialisation from a Composer's Perspective
}

\author{
Hans Timmermans, MSc, MMus \\ HKU - Utrecht University of the Arts, \\ Music and Technology \\ hans.timmermans@hku.nl
}

\begin{abstract}
Multi-channel sound spatialisation is an important aspect of composition, production and performance of electroacoustic music. A multi-speaker set up is seen as a complex instrument on which a composition could be performed. From this composer's perspective the author proposes a set of composition techniques using coded presets and advanced interpolation techniques combined with a design in which sounds in a mix are individual sound objects with individual positions, trajectories, envelopes and acoustic environmental properties. This approach is implemented in cppTK, a scriptable composition and production environment and language for OSX as developed by the author and coded in $\mathrm{C}++$. Despite of the technical implementation of the proposed composition techniques this paper is about composition, not about technology as such. Spatialisation techniques as proposed in this paper should be seen as composition, orchestration and instrumentation techniques rather than technologies to reproduce a recorded reality.
\end{abstract}

\section{INTRODUCTION}

Performing electroacoustic music in a concert often involves multi-channel reproduction with loudspeakers. A multi-channel speaker setup of a specific concert is for most composers just an instrument to perform their composition on. The composition in most cases is not a recorded reality that needs technical perfection in the reproduction on $\mathrm{N}$ loudspeakers, but a composed and designed soundworld that needs a good or, if possible, a perfect instrument. To deal with imperfections of the speaker setups (instruments), the shortage of time to do soundchecks and to calibrate speaker setups (not to mention other obstacles found in general concert practices) a composer might use specific composition techniques and design his workflow to deal with these practicalities.

Copyright: (C) 2015 H. Timmermans. This is an open-access article distributed under the terms of the Creative Commons Attribution License 3.0 Unported, which permits unrestricted use, distribution, and reproduction in any medium, provided the original author and source are credited.
An overview of actual concert practices was given by $\mathrm{N}$. Peters et. al [1], their paper mentions most of the struggles composers have to deal with, this paper sketches composition, orchestration and instrumentation techniques and a workflow in that sense.

Over the last 10 years the notion of a description of sound scenes independent of the actual multi-speaker setup is developing, SPATDIF being a good example. This paper is inspired by that development. [2][3][4]

The author has a background in physics as well as in composition of electronic art music and learned to live with two very different perspectives on 'perfection'. In his compositional work the lack of composition and production tools matching his workflow lead to the development of his own software tools, which are bundled into cppTK, a toolkit with over 600 modules implementing most of the important techniques he needs in composing electronic art music.

The proposed techniques have been implemented in cppTK. cppTK is downloadable and the use of cppTK is partly documented. cppTK is mentioned in this paper but not fully described. The description wouldn't fit in a paper like this, so publishing of cppTK and documenting the use is on the author's website [5].

In the authors workflow a sound in a composition is an object with its own individual properties including spatial properties. Sounds are recorded, created or modified and used as sounding objects in a multi-channel mix. The system as implemented in cppTK facilitates the concept of $\mathrm{N}$ loudspeakers as $\mathrm{N}$ individual instruments but also the concept of $\mathrm{N}$ holes in a wall surrounding the listener and thus immersing the listener in a soundworld or composition. cppTK renders a composition offline into a N-channel audio-file that can easily be tuned to the specifics of a given concert venue.

A composition in this workflow is a time-based script written in a simple c-like scripting-language in which sounds and musical structures are coded. The script describes sounds, (temporal) properties, timing, musical struc- 
tures, pitches, transpositions, envelopes, spatial trajectories etc. as well as synthesizers, algorithms, gestures, analysisand synthesis methods.

Variables in this script can hold different types of data, they can be grouped in series; series can be used with serial composition techniques. Variables may be used to describe trajectories, spatial distributions, artificial acoustics etc.

cppTK implements (among many others) three important techniques to place a soundfile in a multi channel mix.

The first technique is the use of PLAYSOUND(..)

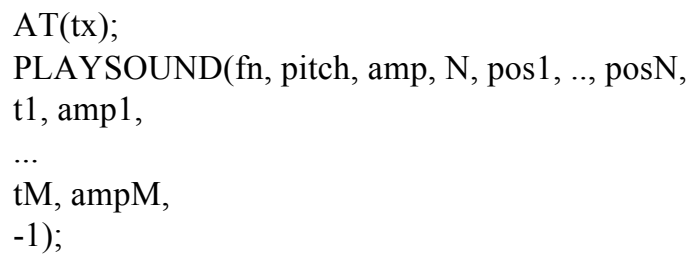

will put the soundfile fn in the mix on time tx. pitch is scaled like MIDI, 60 is no transposition. pitch has no grid, so 67.019550 will be very close to a transposition of a perfect fifth.

amp is in $\mathrm{dB}$, the final mix will be normalized, so amp is relative to other amplitudes in the script.

$\mathrm{N}$ is the number of channels of the soundfile, pos1 to pos $\mathrm{N}$ are coded positions in the multi-channel mix based on multi-speaker diffusion and equal power panning, as explained later in this paper.

AT(tx) will set the timeStamp of the next posted event, several other methods to manage time exist in cppTK. [6]

$<\mathrm{t} 1$, amp1 $>$ to $<\mathrm{tM}$, ampM $>$ describe an envelope, $\mathrm{t} 1$ to $\mathrm{tM}$ are time intervals measured in msec since the start of the sound, amp1 to ampM are relative amplitudes compared to amp.

-1 ends the multi-segment envelope, the number of segments is not limited other than by available computer memory.

Positions pos 1 to posN are just variables and could be named "frontLeft", "left", "crossLeft", "rightBackUp" etc. thus leaving the actual coding into technical parameters for a later stage. Positions could also be generated by designed random processes, serial techniques etc.

The second technique to place a soundfile in a multi channel mix is the use of MOVET(..)

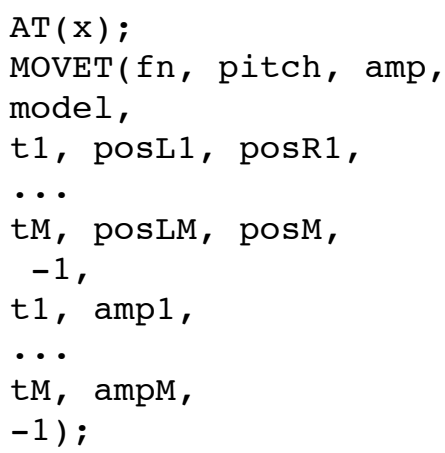

fn, pitch and amp are as described with PLAYSOUND, but MOVET is for stereo soundfiles only.

model is a parameter describing the following trajectory as one shot $($ model $=0)$ or as a repeating trajectory

(model $=1)$.

The trajectory is given as $<\mathrm{t} 1$, posL1, posR $1>$ to $<\mathrm{tM}$, posLM, posRM>, -1 is ending the trajectory. $t 1$ to $\mathrm{tM}$ are measured in msec since the start of the soundfile.

posL.. and posR.. are coded presets.

MOVET will generate and post interpolated values for panning on intervals of $5 \mathrm{msec}$ or longer with a maximum of 40 intermediate values, thus creating a smooth trajectory inbetween coded positions.

An example of a trajectory of a stereo signal in an 8channel space is

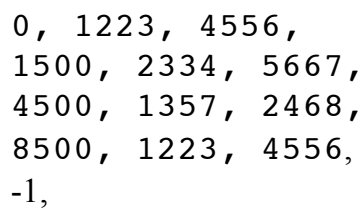

These coded positions are explained in 2 .

A third method is the use of MTCONV(fn, pitch, amp, IR, pIR, vIR, ...);

This implements convolution techniques and is explained in 2.3 on acoustics and convolution.

\section{THEORY}

\subsection{Basics}

As an example of multi channel spatialisation an ideal 8channel audio system with loudspeakers surrounding the audience will be used. It should be noticed that in actual concert practice the ideal set-up sketched in figure 1 is seldom found!

Concerts might be in churches, theatres and factory-halls, open-air, just to name a few 'standard' practices. 
An ideal set-up could be a found in a listening room at an ICMC, in a composition studio or at a laboratory.

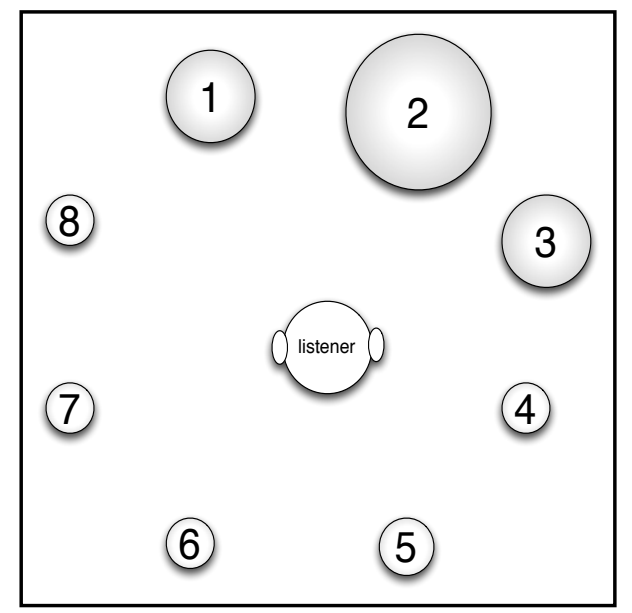

Figure 1. Position code 1223

The concept of 8 loudspeakers being 8 individual instruments or voices is easily coded as " 1 " or " 2 " etc., it is like connecting a sound object to the appropriate system channel / mixer thus implementing a channel based concept.

The concept of 8 holes in a wall transferring the surrounding sound world to the listener is achieved by connecting each individual sound object to all 8 channels with an individual set of 8 volume parameters controlling the perceived spatial position.

"1223" means an emphasis on speaker 2, "12223" is a stronger emphasis on 2 . The codes are translated and finetuned using look-up tables, interpolation and mapping and equal power panning in the end of the calculation. Figure 1 sketches this situation, speaker 2 gets most of the output, speakers 1 and 3 widen the soundobject thus enlarging the sweet spot.

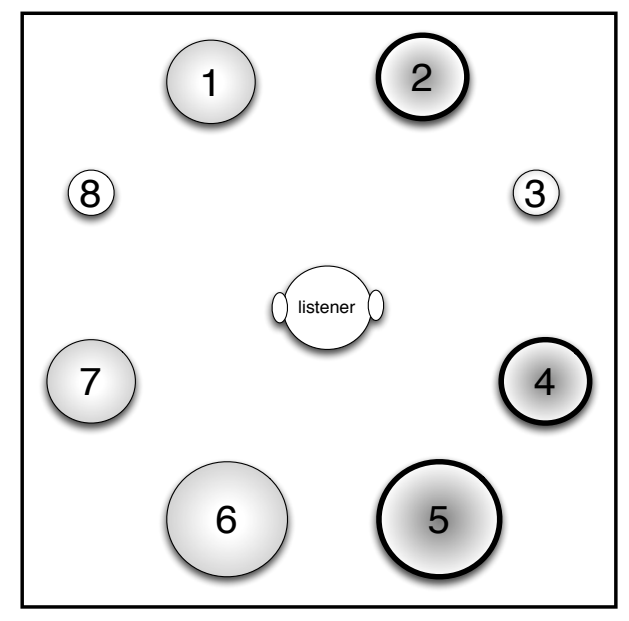

Figure 2. Left channel on 1, right channel on 2, convolution or reverb on 766,554 .
Using a convolution reverb and placing the reverb in the surrounding soundworld adds up to immersive capacities of this concept.

Figure 2 sketches a sound in front of the listener and a calculated (convolution) reverb surrounding the listener in the rear. The convolution might use an impulse response of an real or simulated space but could be another sound as well. The convolution is normalized before it is used in the mix, overdrive caused by resonances is thus prevented.

The system is not developed to reproduce a recorded physical reality or to place point sources in a space. Most of the sounds used in the compositions by the author are stereo sounds or sounds with uncorrelated or weakly correlated channels. One of the advantages of the system is the possibility to use many loudspeakers to diffuse an individual sound thus enlarging the sweet spot and the immersive qualities of the sounds.

\subsection{The panning algorithm}

A position code like " 122233 " will be split as follows: [1][222][33], channel numbers will be counted resulting in: (Examples in pseudo-code)

$v_{1}=1, v_{2}=3, v_{3}=2, v_{4 . . \mathrm{N}}=0$

These values for each of the channels are mapped using a table with fine-tunings and an exponent resulting in relative amplitudes $A_{i}=\operatorname{map}\left[v_{i}\right]^{\propto}$ where $\operatorname{map}[$ index $]$ is an array with fine-tuning data and $\propto$ is a parameter controlling the emphasis on extreme positions.

The total power is calculated and all relative amplitudes are corrected using equal power panning.

The default value of $\propto$ is $1.0, \propto<1.0$ will emphasize the intermediate positions thus shrinking the spatial image (pushing the spatial image to the middle), $\alpha>1.0$ will widen the spatial image (pushing the spatial image to the extremes).

$\operatorname{map}\left[v_{i}\right]$ has default values which can be changed from the script, default values are

$[0,1.38,1.88,2.34,2.91,3.2,4.19]$

These values have been found by experimenting and they supply at least 19 recognizable separate positions inbetween two loudspeakers, which is good enough for the spatial distribution of acousmatic music in a general concert venue with 8 loudspeakers. One of the benefits of this technology is that it works with many different speaker-setups without difficult and time-consuming soundchecks.

Values for $\operatorname{map}\left[v_{i}\right]$ and $\propto$ can be set by the script used to render the mix / multi-channel file(s). As every composition 
is a script to be rendered, a specific version for a concert with specific acoustic issues could easily be rendered, thus supplying convenient flexibility.

\subsection{Acoustics and convolution}

To facilitate the composition, design and production of the combined sound and its acoustic environment the author developed MTCONV(...) a convolution module with some extra's.

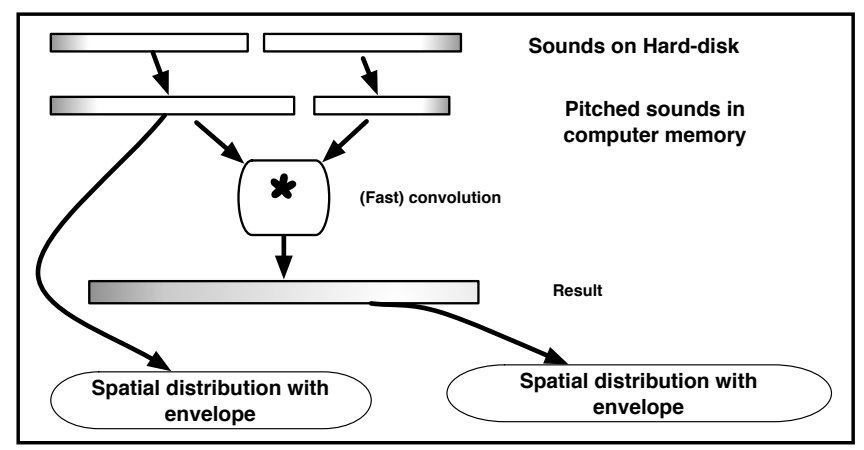

Figure 3. Convolution as a composition tool, MTCONV(..)

Two sounds are put in computer-memory and pitched as if they were played into memory with a sample player. The pitched sounds are convoluted using fast convolution (multiplication in the frequency-domain). The result is normalized. Both the pitched original and the result of the convolution are placed in the multi-channel mix using the panning mechanism as in described before. Thus every sound used in a mix could have its own surrounding acoustic environmental properties, but composers might like to use specific sounds, not necessarily being impulse responses of real spaces, to generate modifications of sound that match artistic aims e.g. sound modification, coloring, hybrid sounds, morphs etc. The fast convolution uses segmented overlapadd convolution if this is speeding up the rendering of the sound (e.g. using long sounds and short impulse responses).

Normalizing the convolution result before it is distributed in space is necessary in cases where the reverb, impulse response or sound has strong formants or resonances.

The convolution algorithm itself is a modified implementation of the overlap-add method as discussed in Numerical Recipes in $\mathrm{C}++$. [7]

An example of the use could be:

$$
\begin{aligned}
& \mathrm{fn}=\operatorname{LOAD}(\text { (“aNiceSound.aif”); } \\
& \text { pitch = 60; amp = 100; } \\
& \text { IR = DIRECTLOAD(“Nicolai.aif”); } \\
& \text { pIR = 60; ampIR = 106; } \\
& \text { low }=0 ; \text { high }=1 ;
\end{aligned}
$$

\author{
$\mathrm{NCH}=2$; \\ MTCONV(fn, pitch, amp, \\ IR, pIR, ampIR, low, high, \\ $\mathrm{NCH}, 1,2,766,554,-1,-1)$;
}

\section{4 cppTK, basics and implementation}

cppTK is a toolkit with well over 600 modules, it runs on Apple's OSX (Yosemite) as a terminal-app. [5]

It acts as a scriptable operating system were individual modules are activated and sound files are opened or loaded from a script. The script is time-based, it is designed to place events in a time-based queue, internally a scheduler is running with a timing precision of one audio-sample. cppTK reads scripts (text files), audio files and many other types of data. It writes and generates scripts, audio files and many other types of data. Resulting audio is a 2-80 tracks audio-file (.aiff) or 2-80 separate mono files, sample rates from $20 \mathrm{k} 05-192 \mathrm{k}$, audio resolution 16 or 24 bits.

\section{CONCLUSION}

The proposed techniques have been used in 10 compositions [8-12] and in many concerts and interactive installations with 2 to 24 loudspeakers. Soundchecks are easy to do; the quality of immersion meets the artistic standards of the author. The best way to understand the techniques sketched in this paper is to listen to a demonstration.

\section{REFERENCES}

[1] N. Peters, G. Marentakis and S. McAdams. Current Technologies and Compositional Practices for Spatialization: A Qualitative and Quantitative Analysis. Computer Music Journal. 35:1, pages 10-27

[2] N. Peters, T. Lossius, J. Schacher, P. Baltazar, C. Bascou, and T. Place. A stratified approach for sound spatialization. Presented at the SMC 2009, July 2009

[3] M. Geier and S. Spors. ASDF: Audio Scene Description Format. Proceedings of the International Computer Music Conference, Belfast, N-Ireland, 2008

[4] Nils Peters, Trond Lossius, and Jan C. Schacher. SpatDIF: Principles, Specification, and Examples. Computer Music Journal. 37:1, pages 11-22

[5] http://www. hanstimmermans.eu/cpptk.html

[6] http://www.hanstimmermans.eu/timing-examples.html

[7] William H. Press, Saul A. Teukolsky, William T. Vetterling, Brian P. Flannery. Numerical Recipes in $\mathrm{C}++$ (third edition). Cambridge University Press. Pages 640 - 647.

[8] "DUST" at ICMC 2006 New Orleans, USA

[9] “AETHER" at ICMC 2009 Montreal, Canada

[10] "ZV02" at ICMC 2011 Ljubljana, Slovenia

[11] "BOQ", "WORDS", "WHISPER" at November Music 2011 Den Bosch, Netherlands.

[12] "BOWL", "Rondvis", "ZAGREB", "PASSION" in other concerts. 